\title{
Shear rate-dependent thrombosis/fibrinolysis tests using non-anticoagulated blood could be useful in the prevention of thrombotic disorders
}

\author{
Junichiro Yamamoto*,1, Yoshinobu ljiri², Muneshige Shimizu³, Shinji Yamamoto ${ }^{4}$, Hideo \\ Ikarugi $^{5}$ \& Kjell S Sakariassen ${ }^{6}$ \\ ${ }^{1}$ Kobe Gakuin University (Professor Emeritus), Kobe, Japan \\ ${ }^{2}$ Faculty of Health \& Nutrition, Osaka Shoin Women's University, Osaka, Japan \\ ${ }^{3}$ Otsuka Pharmaceutical Company, Saga, Japan \\ ${ }^{4}$ Faculty of Sport Sciences, Nihon Fukushi University, Aichi, Japan \\ ${ }^{5}$ School of Economics, University of Hyogo, Kobe, Japan \\ ${ }^{6}$ Biella, BI, Italy \\ *Author for correspondence: yamamoto@nutr.kobegakuin.ac.jp
}

\section{"Prevention of thrombotic disorders such as cardiovascular disease and stroke is an urgent and important task for society"}

First draft submitted: 30 September 2018; Accepted for publication: 16 October 2018; Published online: 19 November 2018

Keywords: diet • exercise $\bullet$ fibrinolysis $\bullet$ native blood $\bullet$ platelet function $\bullet$ shear rate-dependent thrombosis $\bullet$ thrombolysis $\bullet$ thrombus

Prevention of thrombotic disorders such as cardiovascular disease and stroke is an urgent and important task for society. Prevention by suitable diet and exercise is recommended by government guidelines in many countries. In order to get useful and practical results by the recommendations, tests employed to assess the thrombotic status of patients, their quality of diet and their exercise levels have conclusive importance.

Assessment of thrombotic status has been performed over the years by quantifying thrombotic factors and by measuring function using anticoagulated blood samples. However, these approaches do not seem to be successful in assessing thrombotic status. This may be due in part to the belief that adding calcium to anticoagulated blood can restore properties of the original native blood and that individual quantification of thrombotic factors can reflect properties of overall multifactorial native (non-anticoagulated) blood. However, this assumption is wrong and needs to change, not least in particular because relevant in vivo wall shear rates are not included in these tests.

A different approach based on physiological (functional) and biological (evolutionary) ideas was proposed by separate groups in the 1970s. The first group was made up of Baumgartner, Sakariassen and colleagues [1-5], and the other of Kovacs and colleagues [6-10]. Both used native blood as samples (ex vivo) and thrombosis was measured at various shear rates. The Baumgartner, Sakariassen and colleagues thrombosis tests are triggered by various thrombogenic surfaces, including human arterial subendothelium, human fibrillar collagen and human tissue factor/phospholipids at wall shear rates varying from 100 to $32,000 \mathrm{~s}^{-1}$. Blood is drawn directly from an antecubital vein over the prothrombotic surface at various controlled-wall shear rates, thus avoiding coagulation and platelet activation before reaching the prothrombotic surface [4]. These tests have been successfully used in academic and biopharma research since the 1970s. They are used in drug discovery and development and in therapeutic procedures, including optimization of antiplatelet agents and anticoagulation at various wall shear rates [3]. In the Global Thrombosis Tests developed by Kovacs and colleagues, thrombus formation is triggered by high shear stress.

A thrombus is formed by the interaction between blood and blood vessels under blood flow (Virchow's triad). Yamamoto et al. introduced the helium-neon laser-induced in vivo thrombosis system established by Kovacs et al. Subsequently they began research with shear-induced thrombosis/thrombolysis (fibrinolysis) tests both in animals and humans using native blood (ex vivo). Yamamoto and colleagues have analyzed the matching results obtained 
by ex vivo and in vivo tests in animal experiments. Ex vivo and in vivo results were closely correlated, although with exception in rodents with severe endothelial dysfunction, recommending a simultaneous endothelial function test (flow-mediated vasodilation test) [11]. No correlation was found between the results obtained by testing diabetics and patients after stroke with a shear-induced thrombosis test using native blood (thrombotic status analyser) and conventional agonist-induced platelet aggregation measurement using anticoagulated blood [12].

Yamamoto et al. observed qualitative differences between fruit and vegetable varieties using shear-induced thrombosis/fibrinolysis ex vivo tests and He-Ne laser-induced thrombosis in vivo test. They demonstrated that the antithrombotic activity of fruits and vegetables varies within the same species, that is, there are varieties with antithrombotic activity, those with prothrombotic activity and those with neither effect [13]. In healthy volunteers, antithrombotic status was observed within $2 \mathrm{~h}$ of oral administration of an antithrombotic vegetable variety [14]. Further to this, a daily intake of antithrombotic fruit and vegetable varieties over 12 weeks significantly lowered the thrombotic status of humans [15]. Yamamoto and colleagues proposed that the exercise paradox could be avoided by assessing individual thrombotic status with shear-induced thrombosis/fibrinolysis tests [16]. Similar results have been demonstrated by Sakariassen and colleagues in studies focusing on diet supplement, physical exercise and cigarette smoking $[3,17,18]$.

The ex vivo Global Thrombosis Test (GTT) is based on the principles of flow chamber techniques, first described by Baumgartner and Sakariassen, and their colleagues [1,4]. The usefulness of the GTT has already been proven in healthy volunteers [19-21] and in patients [22-25]. Tests using native blood have not been widely used in clinical settings. GTT was first described in 2003 [9] and the first clinical experience with the technique was published by Nishida et al. [26]. GTT has been cited in several review articles [27-29]. The slow increase in the number of publications on GTT might be due to the fast coagulation of non-anticoagulated blood, and hence the concern that tests using native blood might not be suitable to assess thrombotic status in clinical settings. Coagulation is, however, slower under the flow conditions in GTT. Previous studies by Baumgartner, Sakariassen, Kovacs and respective colleagues successfully measuring thrombus formation and resolution provided evidence that the use of native blood under flow conditions is superior to studies using anticoagulated blood. As the variability of test results depends on the technique used to obtain the blood sample, there was concern of high variation regarding test results obtained by GTT. However, reproducibility of the GTT has been tested and published [30]. If blood sampling is performed by a trained operator, the reproducibility of both thrombotic (OT) and thrombolytic activity (LT) is good (the intra-assay coefficient of variation of OT was 10\% and 6\% for LT; the inter-assay coefficient of variation of OT was $8 \%$ and $9 \%$ for LT), which allows for credible statistical analysis of the findings. The point-of-care GTT has proven to be sensitive in detecting small differences in thrombotic and fibrinolytic activities. GTT surpassed the sensitivity of routine coagulation tests such as the prothrombin time test and the activated partial thromboplastin time test, especially in monitoring oral anticoagulants [31]. GTT detected hyper thrombotic status after overwork, which could not be detected by conventional coagulation tests [32]. Shear-dependent thrombosis tests, including the Baumgartner, Sakariassen and colleagues' shear dependent tests and the point-of-care ex vivo Global Thrombosis Test, could be useful in assessing thrombotic status and bleeding of patients, in developing antithrombotic drugs and diets and in proposing antithrombotic programs utlilizing physical exercise for the prevention of thrombotic disorders.

Financial \& competing interests disclosure

The authors have no relevant affiliations or financial involvement with any organization or entity with a financial interest in or financial conflict with the subject matter or materials discussed in the manuscript. This includes employment, consultancies, honoraria, stock ownership or options, expert testimony, grants or patents received or pending, or royalties.

No writing assistance was utilized in the production of this manuscript.

Author contributions

These authors contributed equally to this manuscript. 


\section{References}

1. Baumgartner HR, Turitto VT, Weiss HJ. Effect of shear rate on platelet interaction with subendothelium in citrated and native blood. II. Relationships among platelet adhesion, thrombus dimensions, and fibrin formation. J. Lab. Clin. Med. 95(2), 208-221 (1980).

2. Sakariassen KS, Aarts PA, de Groot PG, Houdijk WP, Sixma JJ. A perfusion chamber developed to investigate platelet interaction in flowing blood with human vessel wall cells, their extracellular matrix and purified components. J. Lab. Clin. Med. 102(4), 522-535 (1983).

3. Sakariassen KS, Turitto VT, Baumgartner HR. Recollections of the development of flow devices for studying mechanisms of hemostasis and thrombosis in flowing whole blood. J. Thromb. Haemost. 2(10), 1681-1690 (2004).

4. Sakariassen KS, Orning L, Turitto VT. The impact of blood shear rate on arterial thrombus formation. Future Sci. OA 1(4), FSO30 (2015).

5. Barstad RM, Roald HE, Cui Y, Turitto VT, Sakariassen KS. A perfusion chamber developed to investigate thrombus formation and shear profiles in flowing native human blood at the apex of well-defined stenoses. Arterioscler. Thromb.. 14(12), 1984-1991 (1994).

6. Görög P, Kovacs IB. Coagulation of flowing native blood: advantages over stagnant (tube) clotting tests. Thromb. Res. 64(5), 611-619 (1991).

7. Ratnatunga CP, Edmondson SF, Rees GM, Kovacs IB. High-dose aspirin inhibits shear-induced platelet reaction involving thrombin generation. Circulation 85(3), 1077-1082 (1992).

8. Gorog DA, Kovacs IB. Thrombotic status analyser: measurement of platelet-rich thrombus formation and lysis in native blood. Thromb. Haemost. 73(3), 514-520 (1995).

9. Yamamoto J, Yamashita T, Ikarugi $\mathrm{H}$ et al. Görög Thrombosis Test: a global in vitro test of platelet function and thrombolysis. Blood Coagul. Fibrinolysis 14(1), 31-39 (2003).

10. Yamamoto J, Inoue N, Otsui K, Ishii H, Gorog DA. Global Thrombosis Test (GTT) can detect major determinants of haemostasis including platelet reactivity, endogenous fibrinolytic and thrombin-generating potential. Thromb. Res. 133(5), 919-926 (2014).

11. Yamamoto J, Tamura Y, Ijiri Y, Iwasaki M, Murakami M, Matsuo O. Evaluation of antithrombotic effect: importance of testing components and methodologies. Drug Discov. Ther. 9(4), 258-266 (2015).

12. Nakajima S, Noguchi T, Taka T et al. A global platelet test of thrombosis and thrombolysis detects a pro-thrombotic state in some patients with non-insulin dependent diabetes and in some patients with stroke. Platelets 11(8), 459-466 (2000).

13. Yamamoto J, Ijiri Y, Tamura Y, Iwasaki M, Murakami M, Okada Y. Reevaluation of antithrombotic fruits and vegetables: great variation between varieties. Drug Discov. Ther. 10(3), 129-140 (2016).

14. Naemura A, Ohira H, Ikeda M, Koshikawa K, Ishii H, Yamamoto J. An experimentally antithrombotic strawberry variety is also effective in humans. Pathophysiol. Haemost. Thromb. 35(5), 398-404 (2006).

15. Ijiri Y, Ishii H, Yamamoto J. Diet of fruits and vegetables with experimental antithrombotic effect may be beneficial to humans in the prevention of arterial thrombotic diseases. Int. J. Drug Dev. Res. 8(3), 12-16 (2016).

16. Ikarugi H, Yamamoto J. The exercise paradox may be solved by measuring the overall thrombotic state using native blood. Drug Discov. Ther. 11(1), 15-19 (2017).

17. Cadroy Y, Pillard F, Sakariassen KS, Thalamas C, Boneu B, Riviere D. Strenous but not moderate exercise increases the thrombotic tendency in healthy sendentary male volunteers. J. Appl. Physiol. 93(3), 829-833 (2002).

18. Roald HE, Orvim U, Bakken IJ, Barstad RM, Kierulf P, Sakariassen KS. Modulation of thrombotic responses in moderately stenosed arteries by cigarette smoking and aspirin ingestion. Arteriosclerosis Thromb. 14(4), 617-621 (1994).

19. Ikarugi H, Yamashita T, Aoki R, Ishii H, Kanki K, Yamamoto J. Impaired spontaneous thrombolytic activity in elderly and in habitual smokers, as measured by a new global thrombosis test. Blood Coagul. Fibrinolysis 14(8), 781-784 (2003).

20. Yamashita T, Sato A, Ikarugi $\mathrm{H}$ et al. Significantly reduced spontaneous thrombolytic activity in older men: a possible explanation for the gender differences in risk of acute coronary syndromes. Thromb. Res. 116(2), 127-131 (2005).

21. Suehiro A, Wakabayashi I, Yamashita T, Yamamoto J. Attenuation of spontaneous thrombolytic activity measured by the global thrombosis test in male habitual smokers. J. Thromb. Thrombolysis 37(4), 414-418 (2014).

22. Suehiro A, Wakabayashi I, Uchida K et al. Impaired spontaneous thrombolytic activity measured by global thrombosis test in males with metabolic syndrome. Thromb. Res. 129(4), 499-501 (2012).

23. Taomoto K, Ohnishi $\mathrm{H}$, Kuga Y et al. Platelet function and spontaneous thrombolytic activity of patients with cerebral infarction assessed by the global thrombosis test. Pathophysiol. Haemost. Thromb. 37(1), 43-48 (2010).

24. Christopoulos C, Farag M, Sullivan K, Wellsted D, Gorog DA. Impaired thrombolytic status predicts adverse cardiac events in patients undergoing primary percutaneous coronary intervention. Thromb. Haemost. 117(3), 457-470 (2017).

25. Niespialowska-Steuden M, Markides V, Farag M et al. Catheter ablation for AF improves global thrombotic profile and enhances fibrinolysis. J. Thromb. Thrombolysis. 44(4), 413-426 (2017).

26. Nishida H, Murata M, Miyaki K, Omae K, Watanabe K, Ikeda Y. Gorog Thrombosis Test: analysis of factors influencing occlusive thrombus formation. Blood Coagul. Fibrinolysis. 17(3), 203-207 (2006). 
27. Paniccia R, Priora R, Liotta AA, Abbate R. Platelet function tests: a comparative review. Vasc. Health Risk Manag. 11, 133-148 (2015).

28. Okafor ON, Gorog DA. Endogenous fibrinolysis: an important mediator of thrombus formation and cardiovascular risk. J. Am. Coll. Cardiol. 65(16), 1683-1699 (2015).

29. Gorog DA, Fayad ZA, Fuster V. Arterial thrombus stability: does it matter and can we detect it? J. Am. Coll. Cardiol. 70(16), 2036-2047 (2017).

30. Farag M, Niespialowska-Steuden $\mathrm{M}$, Okafor $\mathrm{O}$ et al. Relative effects of different non-vitamin $\mathrm{K}$ antagonist oral anticoagulants on global thrombotic status in atrial fibrillation. Platelets 27(7), 687-693 (2016).

31. Otsui K, Gorog DA, Yamamoto J et al. Global Thrombosis Test - a possible monitoring system for the effects and safety of dabigatran. Thromb. J. 13, 39 (2015).

32. Otsui K, Yamamoto J, Inoue N. Overwork accelerates thrombotic reaction implications for the pathogenesis of Karoshi. J. Thromb. Thrombolysis 45(2), 222-224 (2017). 